\title{
Intensity-modulated proton and carbon-ion radiation therapy in the management of major salivary gland carcinomas
}

\author{
Qingting Huang ${ }^{1,2,3 \#}$, Weixu Hu${ }^{1,3 \#}$, Jiyi Hu${ }^{1,3}$, Jing Gao ${ }^{1,3}$, Jing Yang ${ }^{1,3}$, Xianxin Qiu ${ }^{1,3}$, Lin Kong ${ }^{2,3}$, \\ Jiade Jay $\mathrm{Lu}^{1,3}$
}

${ }^{1}$ Department of Radiation Oncology, Shanghai Proton and Heavy Ion Center, Shanghai, China; ${ }^{2}$ Department of Radiation Oncology, Shanghai Proton and Heavy Ion Center, Fudan University Cancer Hospital, Shanghai, China; ${ }^{3}$ Shanghai Engineering Research Center of Proton and Heavy Ion Radiation Therapy, Shanghai, China

Contributions: (I) Conception and design: JJ Lu, L Kong; (II) Administrative support: JJ Lu, L Kong; (III) Provision of study materials or patients: Q Huang, W Hu, J Gao; (IV) Collection and assembly of data: Q Huang, W Hu, J Yang, X Qiu; (V) Data analysis and interpretation: Q Huang, W Hu, J Hu; (VI) Manuscript writing: All authors; (VII) Final approval of manuscript: All authors.

\#These authors contributed equally to this work.

Correspondence to: Lin Kong, MD; Jiade Jay Lu, MD, MBA. Shanghai Proton and Heavy Ion Center, 4365 Kangxin Road, Pudong, Shanghai 201321, China. Email: lin.kong@sphic.org.cn; jiade.lu@sphic.org.cn.

Background: Primary major salivary gland carcinomas (SGCs) present with diverse histological types that are known to be largely radioresistant with a high tendency to develop distant metastasis (DM). Photonbased radiotherapy (RT) is limited in terms of its therapeutic effect and toxicities. In view of the physical and biological advantages of intensity-modulated proton and/or carbon-ion radiation therapy, we aimed to evaluate the short-term therapeutic effect and toxicities in patients with major SGCs treated with this form of radiation therapy.

Methods: Between August 2015 and November 2019, a total of 55 consecutive and non-selected major SGC patients who received particle RT at the Shanghai Proton and Heavy Ion Center (SPHIC) were retrospectively analyzed. The 2-year overall survival (OS), progression-free survival (PFS), local-regional recurrence-free survival (LRRFS), and distant metastasis-free survival (DMFS) rates, as well as prognostic factors were analyzed. Additionally, acute and late toxicities were also analyzed.

Results: With a median follow-up time of 24 (range, 6-57) months, the 2-year OS, PFS, LRRFS, and DMFS rates were $91.6 \%, 78.6 \%, 94.2 \%$, and $83.9 \%$, respectively. At the time of this analysis, four patients had developed local or regional recurrence, and seven additional patients had developed DM. Three patients had died due to disease progression, and another patient with recurrence experienced a late Grade 5 event (hemorrhage) at 9 months after re-irradiation with carbon ion and subsequently died. Otherwise, none of the patients had grade 3 or higher treatment-induced acute or late adverse effects except one who developed grade 3 acute mucositis.

Conclusions: Overall, intensity-modulated proton and/or carbon-ion radiation therapy provided satisfactory therapeutic effectiveness in our major SGCs patients with a low incidence of acute and late toxicities.

Keywords: Proton; carbon ion; radiotherapy (RT); major salivary gland carcinomas (major SGCs)

Submitted Nov 24, 2020. Accepted for publication Dec 26, 2020.

doi: 10.21037/atm-20-7988

View this article at: https://dx.doi.org/10.21037/atm-20-7988 


\section{Introduction}

Major salivary gland carcinomas (SGCs), including parotid, submandibular, and sublingual gland carcinoma, account for $3-6 \%$ of all head and neck cancers (1). Tumors of the major salivary glands are rare and heterogenous, including different histological types, such as mucoepidermoid carcinoma (MEC), adenoid cystic carcinoma (ACC), and salivary duct carcinoma, which are mostly considered radioresistant (2).

Surgery is the mainstay of management for resectable major SGCs $(3,4)$. Postoperative radiotherapy (PORT) is often recommended in high-risk patients with adverse prognostic factors such as a positive surgical margin, high histological grade, tumor size and extension (i.e., pT3-4), lymph node involvement, or presence of perineural invasion (PNI). These prognosticators significantly influence tumor control and survival outcome and warrant adjuvant radiotherapy (RT) (5-9). Definitive RT is recommended for unresectable or inoperable major SGC patients $(3,4)$. However, results from photon-based studies show that the 5-year local control (LC) rates are approximately 50\% or less in inoperable salivary gland tumors (3), suggesting that conventional photon-based RT has limitations for the treatment of major SGCs. Neutron radiation appears to be the appropriate therapy for unresectable or inoperable cases with significantly improved LC, but the risk of severe toxicity is remarkably higher than that of photon-based RT (10-12).

There is an increasing interest in the use of particle (e.g., proton and carbon ion) beams in the treatment of SGCs $(4,13,14)$. Particle beam radiotherapy (PBRT) provides favorable physical and biological advantages compared to photon-beam radiation, and has the potential to deliver a higher dose to the tumor region while sparing the normal structures adjacent to the target volume $(15,16)$. Moreover, as a high linear energy transfer (LET) radiation, the relative biological effectiveness (RBE) of carbon ion beams is higher than that of proton and photon beams, indicating that SGCs, which are historically known to be radioresistant, may respond well to it. The use of intensitymodulated proton and/or carbon ion radiotherapy (IMPT or IMCT) in mixed cases with minor and major SGCs has shown a preliminary superiority in improving LC and reducing radiation-induced toxicities in some clinical studies (13,14,17-20). However, considering the differences in specific clinical presentations and the biological behavior of the tumor site of occurrence, the role of IMPT and/or
IMCT exclusively for major SGCs needs investigation.

The Shanghai Proton and Heavy Ion Center (SPHIC) initiated the clinical application of intensity-modulated PBRT using pencil beam scanning (PBS) technology in May of 2015. In this study, we report the outcomes of the use of IMPT and/or IMCT as the adjuvant or definitive treatment of the first cohort of patients with major SGCs. We present the following article in accordance with the STROBE reporting checklist (available at https://atm.amegroups. com/article/view/10.21037/atm-20-7988/rc).

\section{Methods}

\section{Patients and pretreatment evaluation}

Between August 2015 and November 2019, 55 consecutive and non-selected major SGC patients who received PBRT at the SPHIC were retrospectively analyzed. Pretreatment evaluation included a complete history and physical (H\&P) examination, complete blood count (CBC), serum electrolyte panel, renal and hepatic function tests, and enhanced magnetic resonance imaging (MRI) or enhanced computed tomography (CT) (if MRI was contraindicated) of the head and neck region for all patients. Positron emission tomography (PET)/CT or chest CT, bone scan, and abdominal ultrasonography were employed to exclude distant metastasis (DM). All patients were staged based on the American Joint Committee on Cancer (AJCC) staging system and discussed in the institutional multidisciplinary tumor clinic to confirm the indication for PBRT prior to the registration and inclusion in the institutional tumor registry. The study was conducted in accordance with the Declaration of Helsinki (as revised in 2013). This study was approved by the Institution Review Board (IRB) of the Shanghai Proton and Heavy Ion Center, Shanghai, China (No. 201022EXP-01). Individual consent for this retrospective analysis was waived.

\section{Particle beam radiation therapy}

All patients were immobilized with thermoplastic masks in the supine position. Plain CT for simulation with a $1.5-\mathrm{mm}$ slice thickness from the vertex to the inferior margin of the clavicular heads was performed. MRI-CT fusion was applied for the target delineation. The gross tumor volume (GTV) was defined as the tumor discovered on clinical examination or imaging studies for patients without surgery or after incomplete surgical resection. Clinical target volume (CTV) 
for boost (CTV-boost) was defined as the GTV with a 1$3 \mathrm{~mm}$ margin to deliver the prescribed dose to the tumor. The CTV included the CTV-boost or tumor bed (Patients with R0/R1 resection) and suspected subclinical extension based on the clinical high-risk estimation. The planning target volume (PTV) was created based on the CTV by adding $3 \mathrm{~mm}$ in the lateral direction for setup variability and $3-5 \mathrm{~mm}$ in the depth direction for range uncertainty, which was $3.5 \%$.

Patients who had failed one previous course of photonbased radiation were required to provide their old treatment plans. Dose recovery from the previous RT doses was set at $70 \%$ (21). The dose constraints of the organs at risk (OARs) are based on TD5/5 described by Emami et al. except for the optic nerve (D20 $<30 \mathrm{GyE}$ ), brain stem (Dmax $<45$ GyE), spinal cord (Dmax $<30$ GyE), and temporal lobes (V40 <7.66 cc; V50<4.66 cc) which were based on the previous experience from the National Institute of Quantum and Radiation Science (NIQRS) of Japan $(22,23)$.

PBRT was delivered utilizing the Siemens Syngo ${ }^{\circledR}$ planning system (VB13, Siemens health solution, Erlangen, Germany) with PBS technology. The beam arrangement varied based on the target volume geometry, and individual factors such as beam angles and/or patient positioning reproducibility were considered for optimal dosimetry. The planning for all patients used multi-field optimization (MFO) to maximize conformity and to reduce the dose to nearby OARs. The setup accuracy was verified with a daily orthogonal X-ray using bony landmarks as a reference, and verification CT scans were routinely performed on a weekly basis starting from the second week of PBRT to assess any anatomical changes. Recalculation was performed if clinically needed.

\section{Follow-up}

All patients were advised to adhere to our institutional standardized follow-up protocol after the completion of PBRT. The first follow-up visit was scheduled within 46 weeks after the completion of PBRT, then every 3 months during the first two years, every 6 months in the following 3 years, then annually thereafter. MRI of the head and neck area was arranged at each follow-up. PET/CT and other examinations including chest $\mathrm{CT}$, bone scan, and abdominal ultrasonography were performed if clinically indicated.

\section{Statistics}

The duration of survival was calculated from the date of diagnosis until the date of death or the last follow-up. The time to local, regional, or distant failure/progression was measured from the date of the initiation of PBRT until the documented date of failure/progression. Survival rates were calculated by the Kaplan-Meier method, while the Cox proportional hazard analysis method was performed to determine independent predictive factors. All analyses were performed using SPSS statistics software package (version 23.0, Chicago, IL, USA). Acute toxicities (occurring within 3 months after the initiation of PBRT) were evaluated according to the National Cancer Institute Common Terminology Criteria for Adverse Event (CTC.AE version 4.03 ) and late toxicities (occurring $>3$ months after initiation of PBRT) were scored using the Radiotherapy Oncology Group (RTOG) late radiation morbidity scoring system.

\section{Results}

\section{Patient characteristics}

55 consecutive and non-selected major SGC patients who received PBRT at the SPHIC were analyzed between August 2015 and November 2019. The median follow-up was 24 (range, 6-57) months for the entire cohort. Primary sites of the tumors included the parotid gland $(n=37)$, submandibular gland $(\mathrm{n}=17)$, and sublingual gland $(\mathrm{n}=1)$. Histologic types included ACC $(\mathrm{n}=23)$, adenocarcinoma $(n=9)$, MEC $(n=7)$, lymphoepithelial carcinoma $(n=4)$, salivary duct carcinoma $(n=2)$, rhabdomyosarcoma $(n=2)$, acinar cell carcinoma $(n=2)$, and others $(n=6)$. Forty-one patients presented with newly diagnosed major SGCs. The remaining 14 patients presented with local recurrence after previous treatment, including nine who received prior radiation therapy with or without surgery, and five who received surgery without previous radiation. The patients who received previous resection with $\mathrm{R} 0$ or R1 had indicators for adjuvant radiation such as the stage of the disease (T3-T4/N2-3), a positive surgical margin, PNI, or a high-grade pathology type. The characteristics of the patients are summarized in Table 1.

\section{PBRT}

All patients received either IMPT $(n=8)$, IMCT $(n=37)$, or IMPT combined with IMCT ( $\mathrm{n}=10)$ using PBS technology. The nine patients with local recurrence after previous RT were re-irradiated with IMCT (54-63 GyE/18-20 fractions). The remaining five patients with recurrence 
Table 1 Characteristics of the patients, their disease, and treatments

\begin{tabular}{|c|c|}
\hline Characteristic & No. of patients (\%) \\
\hline Median age [range], years & $41[14-92]$ \\
\hline \multicolumn{2}{|l|}{ Gender } \\
\hline Male & $33(60.0)$ \\
\hline Female & $22(40.0)$ \\
\hline \multicolumn{2}{|l|}{ Disease status } \\
\hline Primary & $41(74.5)$ \\
\hline Recurrence & $14(25.5)$ \\
\hline \multicolumn{2}{|l|}{ RT parameters } \\
\hline Initial treatment & $46(83.6)$ \\
\hline Re-radiotherapy & $9(16.4)$ \\
\hline \multicolumn{2}{|l|}{ Primary site } \\
\hline Parotid gland & $37(67.3)$ \\
\hline Submandibular gland & $18(32.7)$ \\
\hline \multicolumn{2}{|l|}{ Histology } \\
\hline Adenoid cystic carcinoma & $23(41.8)$ \\
\hline Adenocarcinoma & $9(16.4)$ \\
\hline Mucoepidermoid carcinoma & $7(12.7)$ \\
\hline Lymphoepithelial carcinoma & $4(7.3)$ \\
\hline Salivary duct carcinoma & $2(3.6)$ \\
\hline Rhabdomyosarcoma & $2(3.6)$ \\
\hline Acinic cell carcinoma & $2(3.6)$ \\
\hline Others & $6(10.9)$ \\
\hline \multicolumn{2}{|l|}{ Surgery status } \\
\hline Ro & $17(30.9)$ \\
\hline $\mathrm{R} 1$ & $10(18.2)$ \\
\hline $\mathrm{R} 2$ & $17(30.9)$ \\
\hline No surgery & $11(20.0)$ \\
\hline \multicolumn{2}{|l|}{ Clinical T classification } \\
\hline TO & $1(1.8)$ \\
\hline $\mathrm{T} 1$ & $9(16.4)$ \\
\hline $\mathrm{T} 2$ & $18(32.7)$ \\
\hline T3 & $10(18.2)$ \\
\hline $\mathrm{T} 4$ & $17(30.9)$ \\
\hline
\end{tabular}

Table 1 (continued)
Table 1 (continued)

\begin{tabular}{lc}
\hline Characteristic & No. of patients $(\%)$ \\
\hline Clinical N classification & $46(83.6)$ \\
N0 & $5(9.1)$ \\
N1 & $4(7.3)$ \\
N2 & \\
Stage & $8(14.5)$ \\
I & $15(27.3)$ \\
II & $11(20.0)$ \\
III & $21(38.2)$ \\
IV & \\
Radiotherapy technique & $8(14.5)$ \\
IMPT & $37(67.3)$ \\
IMCT & $10(18.2)$ \\
IMPT + IMCT &
\end{tabular}

IMPT, intensity-modulated proton radiotherapy; IMCT, intensitymodulated carbon-ion radiotherapy.

received, either adjuvantly or definitively, RT naïve with IMPT (66 GyE/33 fractions, $\mathrm{n}=1$ ) or IMCT (63$69 \mathrm{GyE} / 18-23$ fractions, $\mathrm{n}=2 ; 70 \mathrm{GyE} / 20$ fractions, $\mathrm{n}=2$ ). Fifteen patients with newly diagnosed SGC who achieved R0 resection received IMPT (54-60 GyE/27-30 fractions, $\mathrm{n}=7$ ) or IMCT (60 GyE/20 fractions, $\mathrm{n}=8$ ), respectively. Ten patients completed a combination of IMPT and IMCT boost, and another 16 newly diagnosed patients completed IMCT according to our standard institutional protocols. The details of the dose/fractionation schemes are detailed in Table 2.

\section{Survival outcomes}

Within the median follow-up time of 24 (range, 6-57) months, four patients had died: Two newly diagnosed patients died of lung and bone metastasis 23 and 25 months after a combination of IMPT and IMCT boost, respectively; one re-irradiated patient died of local recurrence 24 months after re-irradiation with IMCT; another patient with recurrence died of grade 5 hemorrhage 9 months after re-irradiation with carbon ion. In addition, four patients developed local or regional recurrence after 
Table 2 Fractionations of the IMPT/IMCT treatment scheme

\begin{tabular}{|c|c|c|c|}
\hline Fractionation & Total dose (GyE) & Fraction (Fx) & No. of patients \\
\hline \multicolumn{4}{|l|}{ IMPT } \\
\hline 66 GyE/33 Fx & 66 & 33 & 1 \\
\hline 60 GyE/30 Fx & 60 & 30 & 3 \\
\hline 56 GyE/28 Fx & 56 & 28 & 3 \\
\hline \multicolumn{4}{|l|}{ IMPT + IMCT } \\
\hline 56 GyE/28 Fx + 18 GyE/6 Fx & 74 & 34 & 1 \\
\hline 56 GyE/28 Fx + 17.5 GyE/5 Fx & 73.5 & 33 & 4 \\
\hline 56 GyE/28 Fx + 15 GyE/5 Fx & 71 & 33 & 5 \\
\hline 69 GyE/23 Fx & 69 & 23 & 1 \\
\hline 66.5 GyE/19 Fx & 66.5 & 19 & 1 \\
\hline 66 GyE/22 Fx & 66 & 22 & 1 \\
\hline 63 GyE/21 Fx & 63 & 21 & 4 \\
\hline 63 GyE/18 Fx & 63 & 18 & 9 \\
\hline 60 GyE/20 Fx & 60 & 20 & 12 \\
\hline $54 \mathrm{GyE} / 18 \mathrm{Fx}$ & 54 & 18 & 1 \\
\hline
\end{tabular}

IMPT, intensity-modulated proton radiotherapy; IMCT, intensity-modulated carbon-ion radiotherapy.

IMCT or a combination of IMPT and IMCT boost, and seven additional patients developed lung, bone, or brain metastases after PBRT. The 2-year overall survival (OS), progression-free survival (PFS), local-regional recurrencefree survival (LRRFS), and distant metastasis-free survival (DMFS) rates were $91.6 \%, 78.6 \%, 94.2 \%$, and $83.9 \%$, respectively, for the entire cohort (Figure 1).

\section{Predictive factors for OS, PFS, LRRFS, DMFS}

The differences in the survival probabilities were compared by the log-rank test in terms of age, gender, disease status (newly diagnosed $v s$. recurrent), RT parameter (RT naïve vs. re-RT), surgery status (R0-1 vs. R2 + no surgery, and N/A), primary site (parotid gland vs. others), histology (ACC vs. others), T classification (T0-2 vs. T3-4), N classification (N0 vs. N1-2), and stage (I-II vs. III-IV) (Table 3). Univariate analysis showed that OS was associated with surgery status $(\mathrm{P}=0.018)$, and $\mathrm{PFS}$ was associated with the surgery status $(\mathrm{P}=0.005)$, $\mathrm{T}$ classification $(\mathrm{P}=0.001)$, and stage $(\mathrm{P}=0.002)$ (Figure 2). The results also showed statistically significant differences in disease status $(\mathrm{P}=0.020)$, RT parameters $(\mathrm{P}=0.045)$, surgery status $(\mathrm{P}=0.021)$, and $\mathrm{T}$ classification $(\mathrm{P}=0.051)$ for LRRFS (Figure 3$)$. In addition, DMFS was significantly associated with the $T$ classification $(\mathrm{P}=0.006), \mathrm{N}$ classification $(\mathrm{P}=0.030)$, and stage $(\mathrm{P}=0.015)$ (Figure 4).

Multivariate analysis using Cox regression identified the predictive factors with a $\mathrm{P}$ value of 0.5 or less from the univariate analyses for PFS and DMFS (Table 4). Multivariate analysis illustrated that surgery status was an independent prognostic factor for PFS. No significant prognostic factor was identified for DMFS in the multivariate analysis.

\section{Acute and late toxicities}

The most commonly observed acute adverse effects included grade 1 or 2 mucositis, dermatitis, and xerostomia: Four patients experienced acute grade 2 mucositis, four 

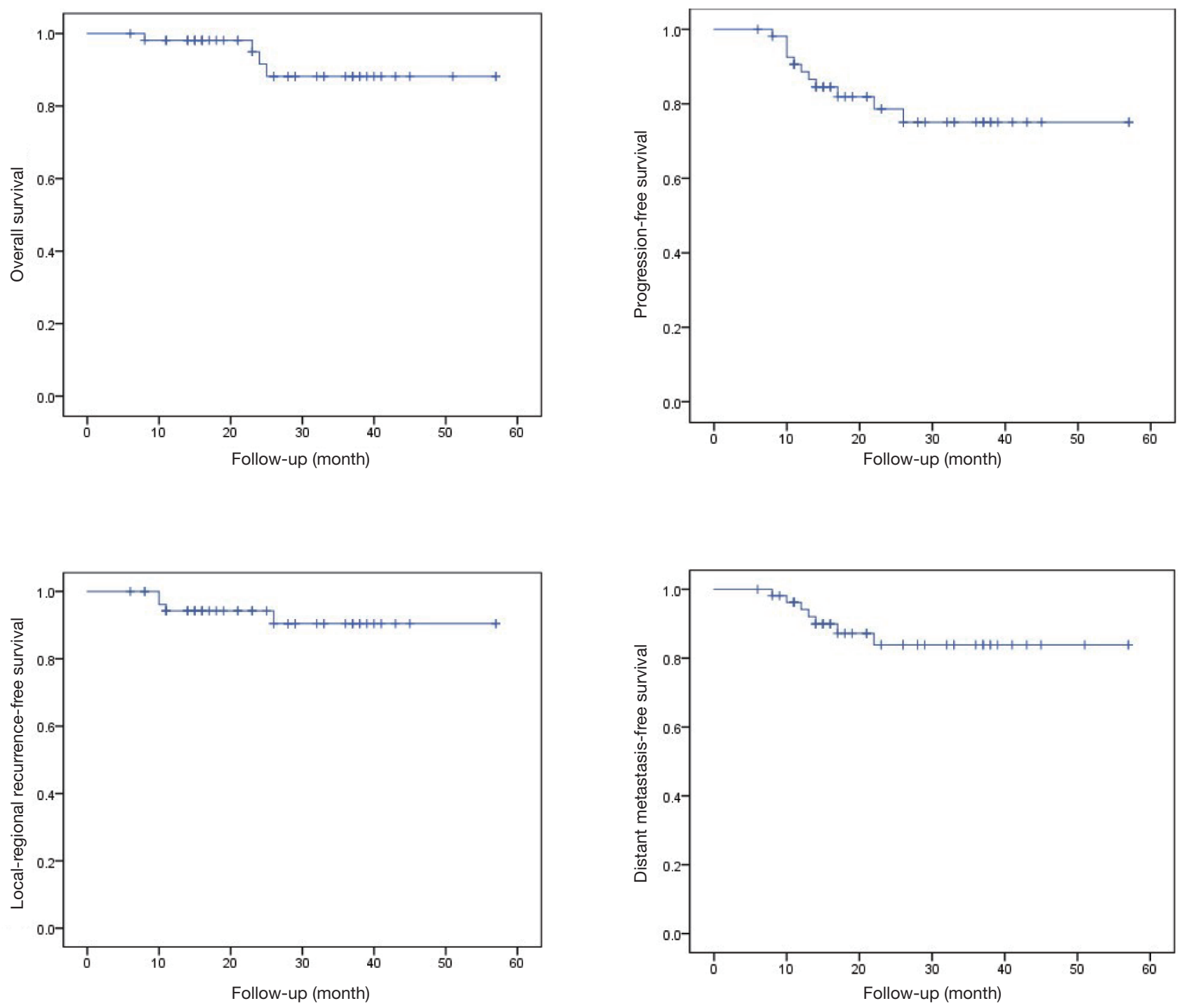

Figure 1 OS, PFS, LRRFS, and DMFS curves of the entire cohort. OS, overall survival; PFS, progression-free survival; LRRFS, localregional recurrence-free survival; DMFS, distant metastasis-free survival.

patients experienced an acute grade 2 dermatitis, and one patient had grade 3 mucositis. No grade 4 or 5 acute toxicities were observed. The most common late side effects included grade 1 xerostomia, hearing impairment, tinnitus, and dysphagia. One patient with recurrent disease died due to hemorrhage at 9 months after re-irradiation with a minimal IMCT dose (54 Gy RBE in 18 fractions) (Table 5).

\section{Discussion}

This study presents 55 consecutive and non-selected patients with major salivary gland malignancies who received adjuvant or radical IMPT and/or IMCT at the SPHIC. With a median follow-up time of 24 (range, 6-57) months, the 2-year OS, PFS, LRRFS and DMFS rates were $91.6 \%, 78.6 \%, 94.2 \%$, and $83.9 \%$, respectively. At the time of this analysis, four patients had developed local or regional recurrence, and seven patients had developed DM. Three patients had died due to disease progression. Another patient with recurrence died following a late grade 5 hemorrhage after re-irradiation with carbon ion beams. Otherwise, none of the patients experienced grade 3 or higher treatment-induced acute or late adverse effects except for 1 (2\%) who developed grade 3 acute 
Table 3 Univariate analysis for the 2-year OS, PFS, LRRFS, DMFS

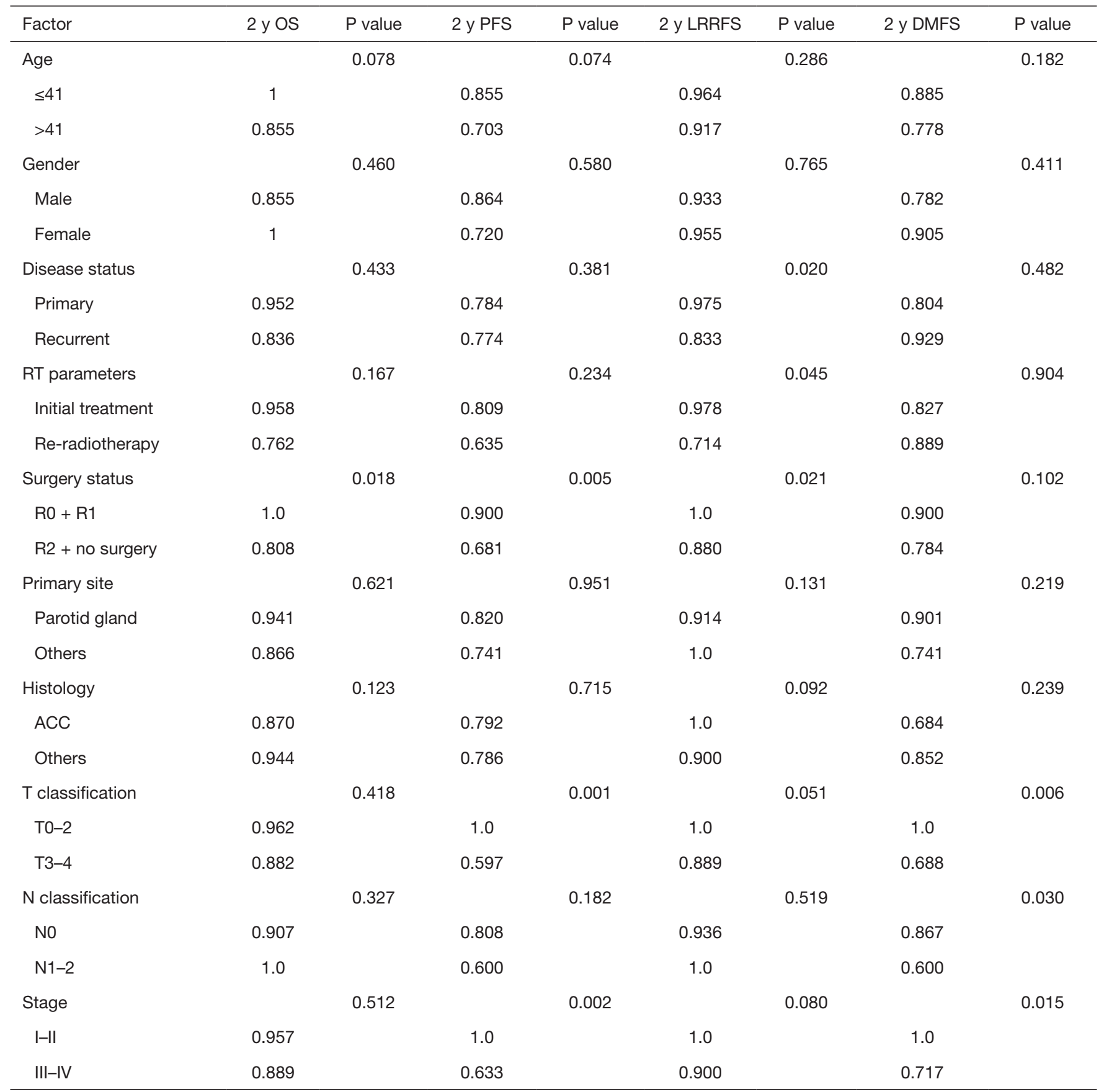

OS, overall survival; PFS, progression-free survival; LRRFS, local-regional recurrence-free survival; DMFS, distant metastasis-free survival; ACC, adenoid cystic carcinoma.

mucositis.

The results for patients with unresectable diseases treated with photon-based RT have shown that the role of conventional RT in the management of SGCs is limited
(24-26). In a study of 64 patients with inoperable tumors, Mendenhall et al. reported that the 5-year OS and 5-year LC were approximately $50 \%$ and $42 \%$, respectively (26). Spratt et al. also demonstrated that the 2-/5-year OS and 

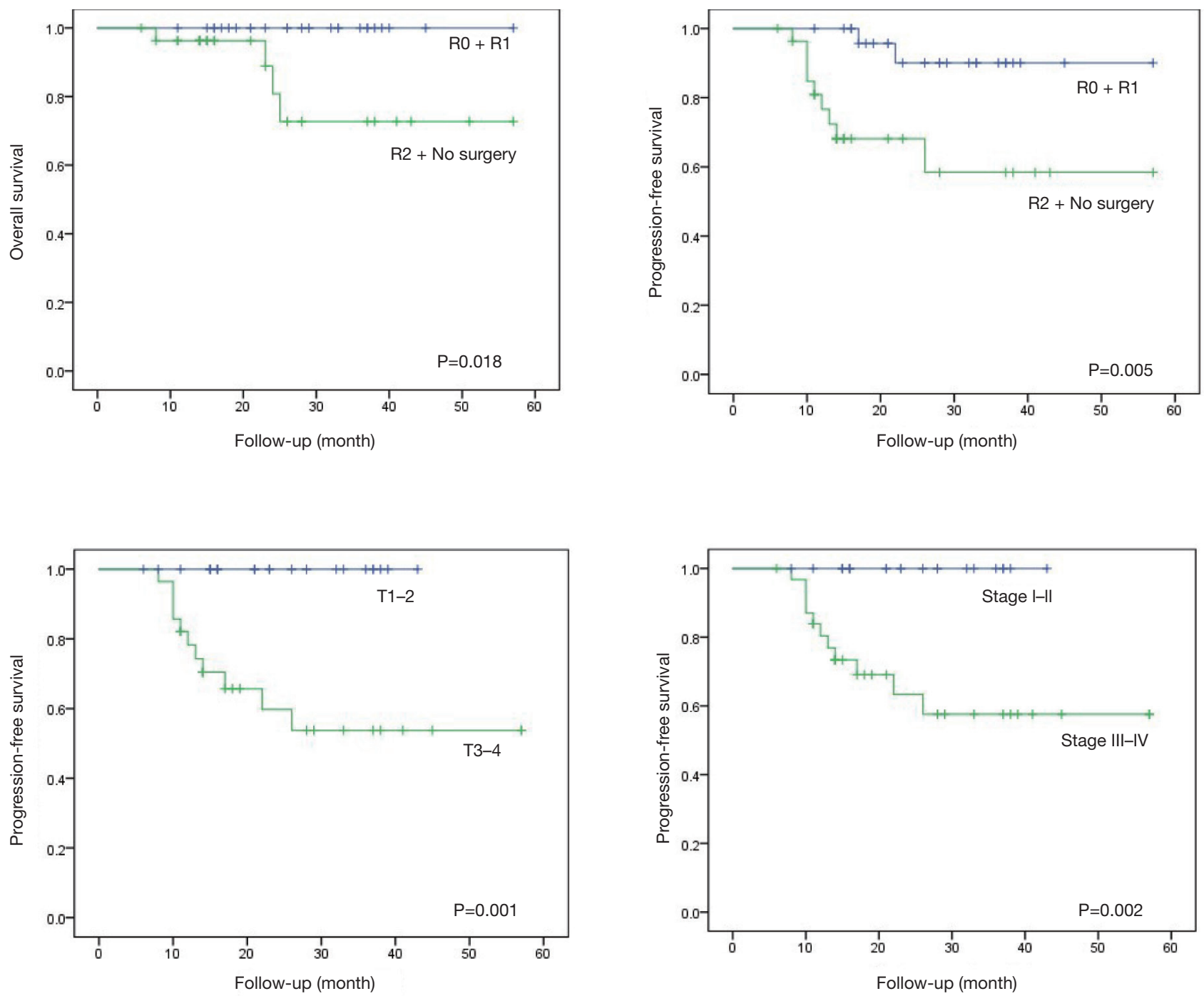

Figure 2 OS curves according to surgery status. PFS curves showing surgery status with R2 and no surgery, T3-4, and stage III-IV diseases had a worse PFS. OS, overall survival; PFS, progression-free survival.

LC were only $50 \% / 29 \%$ and $69 \% / 55 \%$, respectively, in a retrospective study of 27 patients with unresectable salivary gland tumors treated with photon-based RT (25). Conversely, fast neutron RT has provided somewhat more favorable outcomes for those relatively radioresistant tumors compared to photon-based RT $(10,12,27)$. Timoshchuk et al. reported a favorable 2-/5-year LC rate of $88 \% / 78 \%$ and a $2-/ 5$-year OS rate of $82 \% / 62 \%$ using radical neutron RT in treating 145 patients with malignant salivary gland tumors (27), suggesting that neutron, as a high LET radiation, appears to be beneficial in improving OS and LC. However, the incidence of acute and late toxicities caused by neutrons was relatively high. Davis et al. showed that acute xerostomia and mucositis were observed in $89 \%$ and $88 \%$ of patients, respectively, during neutron treatment, and $56 \%$, $52 \%$, and $74 \%$ of patients who received a telephone survey experienced late toxicities of trismus, dysphagia, and saliva decrease, respectively (12). Although the above published clinical studies often contain heterogenous characteristics of patients and diseases (e.g., primary site, tumor pathology, and stages), these results demonstrate that radical RT with either photon or neutron is still limited in terms of its therapeutic effect or toxicities in treating unresectable major SGCs. 

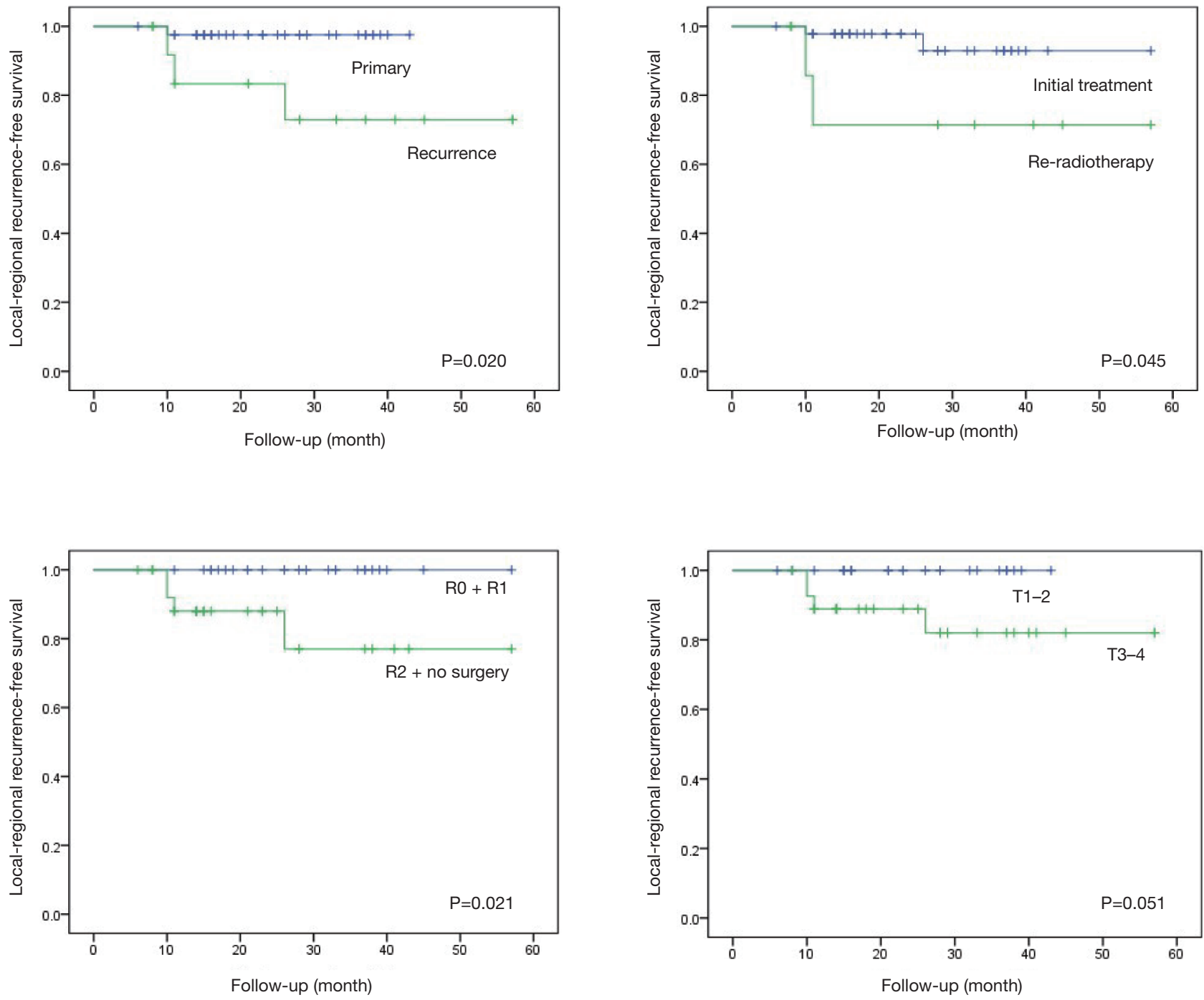

Figure 3 Local-regional recurrence-free survival curves according to disease status, radiotherapy (RT) parameter, surgery status, and T classification.

With respect to resectable diseases, surgery with adjuvant $\mathrm{RT}$ is recommended for patients at high risk of local-regional recurrence (6-9,28-30). In a retrospective study reported by Al-Mamgani et al., 186 patients with parotid gland carcinoma received postoperative photon RT. After a median follow-up of 58 months, the 5 -year OS and LC were $68 \%$ and $89 \%$, respectively (9). Chung et al. reported that the 5 -year OS and LC outcomes were $76 \%$ and $97 \%$, respectively, for patients treated with postoperative photon RT for cancers excluding ACC of the major salivary glands (30). Lee et al. demonstrated that the 5 -year OS of adjuvant photon RT for 1,313 patients with major salivary ACC was $82.4 \%$ (28). These studies indicated that postoperative radiation therapy for major SGCs was associated with an improved therapeutic effect. Our study showed similar findings, and our multivariate analysis showed surgery status was an independent prognostic factor for PFS. However, side effects induced by photon-based PORT may severely compromise the quality of life (QoL) of patients who achieve long-term survival and tumor control. Hosni et al. observed that grade 3 late toxicities occurred in nine patients after adjuvant photon RT, including osteoradionecrosis in four cases, neck fibrosis in three cases, trismus in one 
Page 10 of 14
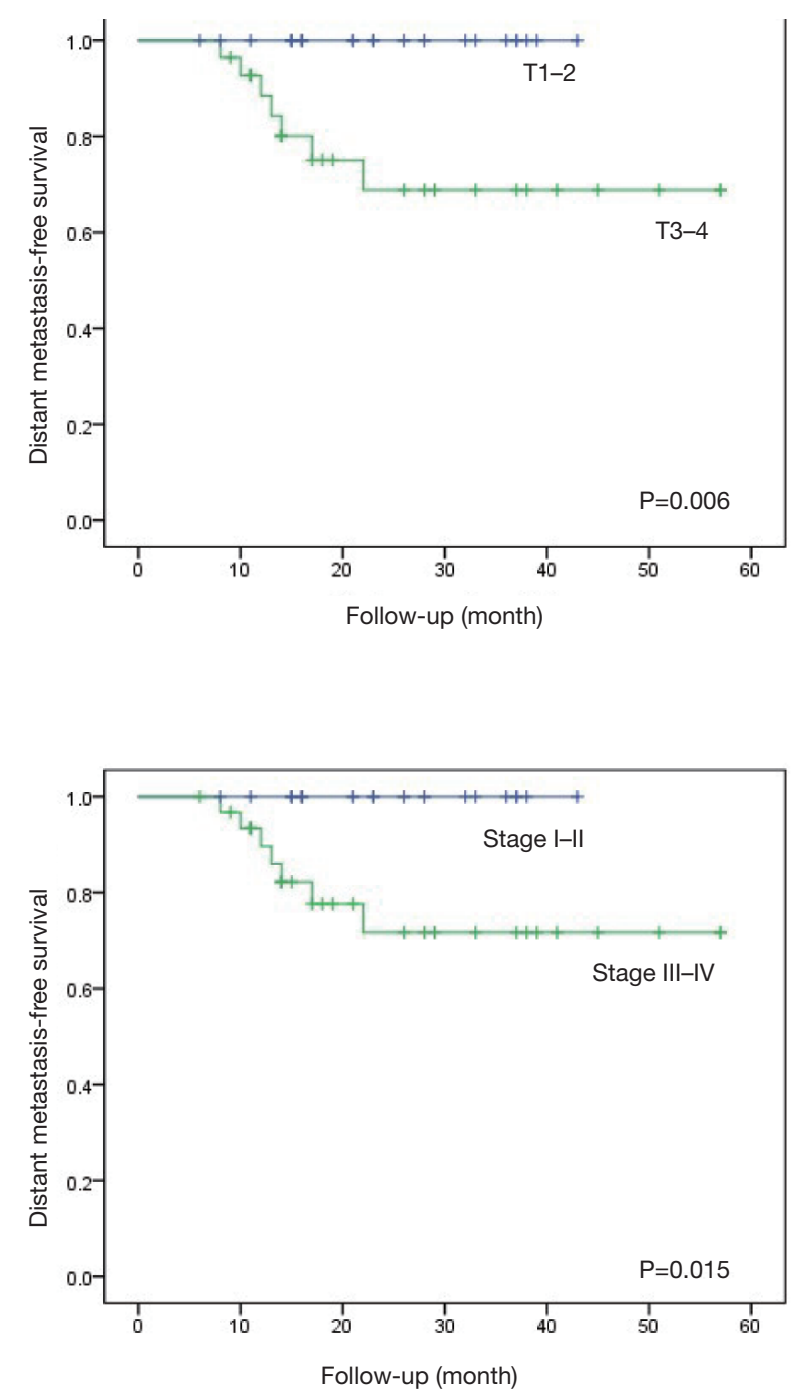

Huang et al. Particle radiotherapy for major salivary gland carcinomas

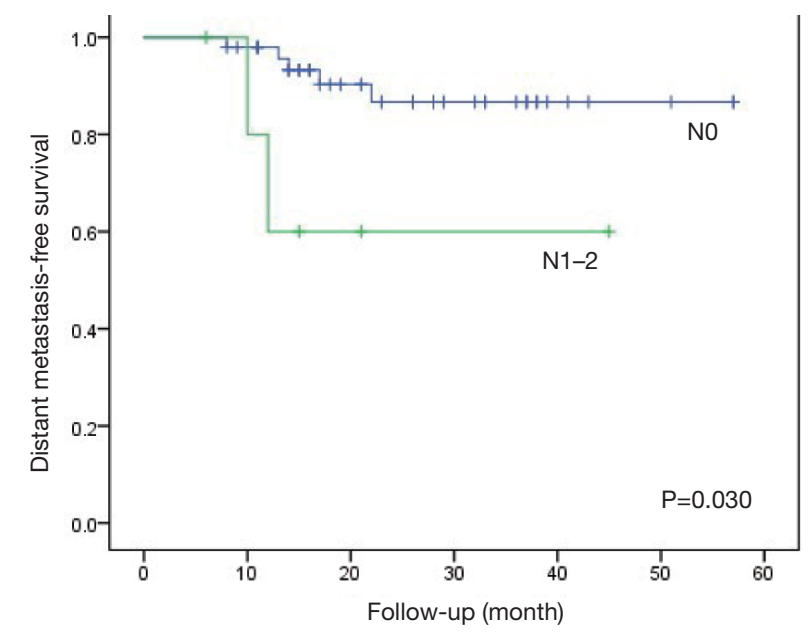

Figure 4 Distant metastasis-free survival curves show that T3-4, N1-2, and stage III-IV diseases had worse DMFS. DMFS, distant metastasis-free survival.

case, and dysphagia in one case (7). Swisher-McClure et al. demonstrated that the incidence of grade $2 / 3$ acute mucositis and dysphagia was $91 \%$ and $27 \%$, respectively, in pediatric patients with parotid gland carcinoma after radical surgery irradiated with adjuvant RT (18). Obviously, RT technology with improved tolerability is more favorable for patients with high risk pathological factors.

Proton and carbon ion beams can provide a more focused dose distribution due to their physical characteristics; therefore, a higher dosage can be delivered to the tumor volume while sparing the adjacent normal organs (15). Moreover, the high RBE of carbon ion has the advantage of destroying these tumors, which were thought to be radioresistant. In a retrospective multicenter study of 69 patients with major SGCs who received carbon-ion radiotherapy (CIRT), Hayashi et al. demonstrated that CIRT can result in good survival and LC rate. The 3-year OS and LC rates were $94 \%$ and $81 \%$, respectively (31). Sulaiman et al. also reported 2-year OS and LC rates of $94 \%$ and $88 \%$, respectively, for ACC of the head and neck (including 35 cases of major salivary gland ACC) utilizing CIRT (14). Koto et al. adopted definitive CIRT for 46 patients with locally advanced parotid gland carcinomas and reported 5 -year LC and OS rates of $74.5 \%$ and $70.1 \%$, respectively (32). Therefore, combined with the results of our study, the therapeutic effect of PBRT for the 
Table 4 Cox proportional hazard regression analysis for 2-year PFS and DMFS

\begin{tabular}{|c|c|c|c|c|}
\hline Factors & \multicolumn{2}{|c|}{ PFS } & \multicolumn{2}{|c|}{ DMFS } \\
\hline Age & 0.30 & $2.29(0.48-10.93)$ & 0.58 & $1.69(0.07-10.72)$ \\
\hline Gender & - & - & 0.87 & $0.86(0.14-5.18)$ \\
\hline Disease status & 0.46 & $0.42(0.04-4.06)$ & - & - \\
\hline Surgery status & 0.01 & $7.69(1.50-39.44)$ & 0.12 & $4.19(0.68-25.87)$ \\
\hline Primary site & 0.69 & $1.32(0.34-5.17)$ & 0.23 & $2.68(0.54-13.36)$ \\
\hline Histology & 0.56 & $1.50(0.38-5.92)$ & 0.84 & $0.85(0.17-4.26)$ \\
\hline T classification & - & - & - & - \\
\hline
\end{tabular}

PFS, progression-free survival; DMFS, distant metastasis-free survival; RT, radiotherapy; HR, hazard ratio; Cl, confidence interval.

Table 5 Type and frequency of adverse events

\begin{tabular}{|c|c|c|c|c|c|}
\hline Toxicity & \multicolumn{5}{|c|}{ Grade, n (\%) } \\
\hline \multicolumn{6}{|l|}{ Acute } \\
\hline Mucositis & $14(25.5)$ & $4(7.3)$ & $1(1.8)$ & 0 & 0 \\
\hline Dermatitis & $29(52.7)$ & $4(7.3)$ & 0 & 0 & 0 \\
\hline Hearing impairment & $4(7.3)$ & 0 & 0 & 0 & 0 \\
\hline Tinnitus & $5(9.1)$ & 0 & 0 & 0 & 0 \\
\hline Dysphagia & $1(1.8)$ & 0 & 0 & 0 & 0 \\
\hline Late & & & & & 0 \\
\hline Tinnitus & $5(9.1)$ & 0 & 0 & 0 & 0 \\
\hline Dysphagia & $4(7.3)$ & 0 & 0 & 0 & 0 \\
\hline Hemorrhage & 0 & 0 & 0 & 0 & $1(1.8)$ \\
\hline Lower cranial neuropathy & $1(1.8)$ & 0 & 0 & 0 & 0 \\
\hline Limited mouth opening & $2(3.6)$ & 0 & 0 & 0 & 0 \\
\hline
\end{tabular}

Toxicities were evaluated according to the CTCAE version 4.03 . 
management of major SGCs appears to be significantly improved compared with photon-based RT.

$\mathrm{DM}$ is the most common pattern of failure in the current study. This finding is consistent with previous reports $(14,31,33,34)$. In a study consisting of 255 patients with major SGCs, 57 (22\%) patients developed DM after radical or adjuvant photon RT within a median follow-up period of 31 months (34). Hsieh et al. also reported that 20 out of 65 patients $(30.1 \%)$ with submandibular gland carcinoma treated with postoperative photon RT developed DM within a median follow-up period of 42 months. DM was also associated with lymph node status (N-classification) and primary size (T-classification) (29). These results are also consistent with our findings, insofar as DM occurred in seven $(13 \%)$ out of 55 of our patients. In addition, our univariate analysis indicated that the N/T classification and stage factors were associated with DMFS. Major SGCs are prone to develop DM, especially in patients with ACC, sarcoma, and nodal involvement. Preclinical research has found that carbon ion and proton have the potential to suppress the capability of malignant tumor cells to metastasize $(35,36)$, and has provided evidence that PBRT may be superior to conventional photon RT in decreasing the rate of DM in clinical settings. However, the potential for an antimetastatic effect using particle beam RT in major SGCs has not been confirmed in our study, and should be further investigated.

None of our patients experienced grade 3 or higher acute toxicity except for 1 (2\%) who developed grade 3 acute mucositis. Only one patient with recurrent disease died of grade 5 hemorrhage after re-irradiation using CIRT to 54 Gy RBE (18 fractions). Romesser et al. compared the acute toxicities of patients with major SGCs after radical surgery, radiation using adjuvant photon, or proton therapy. A total of 41 patients were enrolled in that study, of which 23 patients received photon therapy and the remaining 18 patients received proton beam RT. The incidence of acute side effects in the proton therapy group was remarkably lower than in those who received photonbased RT (grade 2 dysphagia: $5.6 \%$ vs. $65.2 \%, \mathrm{P}<0.001$; grade $2 / 3$ mucositis: $16.7 \%$ vs. $52.2 \%, \mathrm{P}=0.019$ ) (37). In another study, Spratt $e$ al. reported that 13 (48\%) patients experienced acute grade 3 acute toxicities such as mucositis and dysphagia, and three (11\%) patients developed grade 3 late toxicities including dysphagia, mucositis, and hearing loss in unresectable salivary gland tumors after photon RT to radical dose (25). On the contrary, our study showed that only one patient experienced grade 3 acute mucositis, and one additional patient with recurrence with re-irradiation developed massive hemorrhage. Hayashi et al. showed that $14(20.3 \%)$ patients developed grade 3 acute mucositis or dermatitis during CIRT, and $2(3 \%)$ patients developed grade 3 late dysphagia or brain abscess, demonstrating that the incidence and degree of toxicities significantly decreased with proton or carbon ion radiation by virtue of its physical characteristic advantage (31).

Several limitations in the present study need to be addressed. Firstly, our study was a retrospective case series with a relatively small sample size. Given the rarity of major salivary gland neoplasms, most of the published literature on this subject is limited by its retrospective nature and limited number of cases. Secondly, the median followup period of the present study ( 24 months) is relatively short. For certain histology subtypes, especially those with relatively indolent natures, longer follow-up is needed to further assess the long-term outcomes.

\section{Conclusions}

Our results indicated that IMPT and/or IMCT provided satisfactory therapeutic effectiveness in patients with malignancies of the major salivary gland and produced a low incidence of acute and late toxicities. The 2-year OS, PFS, LRRFS, and DMFS rates were 91.6\%, 78.6\%, 94.2\%, and $83.9 \%$, respectively. Long-term follow-up is required to confirm the efficacy and safety of PBRT in the treatment of major salivary gland malignancies.

\section{Acknowledgments}

Funding: This work was mainly supported by the Science and Technology Commission of Shanghai Municipality (grant No. 19411951000).

\section{Footnote}

Reporting Checklist: The authors have completed the STROBE reporting checklist. Available at https://atm. amegroups.com/article/view/10.21037/atm-20-7988/rc

Data Sharing Statement: Available at https://atm.amegroups. com/article/view/10.21037/atm-20-7988/dss

Conflicts of Interest: All authors have completed the ICMJE uniform disclosure form (available at https://atm. amegroups.com/article/view/10.21037/atm-20-7988/coif). The authors have no conflicts of interest to declare. 
Ethical Statement: The authors are accountable for all aspects of the work in ensuring that questions related to the accuracy or integrity of any part of the work are appropriately investigated and resolved. The study was conducted in accordance with the Declaration of Helsinki (as revised in 2013). This study was approved by the Institution Review Board (IRB) of the Shanghai Proton and Heavy Ion Center, Shanghai, China (No. 201022EXP-01). Individual consent for this retrospective analysis was waived.

Open Access Statement: This is an Open Access article distributed in accordance with the Creative Commons Attribution-NonCommercial-NoDerivs 4.0 International License (CC BY-NC-ND 4.0), which permits the noncommercial replication and distribution of the article with the strict proviso that no changes or edits are made and the original work is properly cited (including links to both the formal publication through the relevant DOI and the license). See: https://creativecommons.org/licenses/by-nc-nd/4.0/.

\section{References}

1. Witt RL. Major salivary gland cancer. Surgical Oncology Clinics of North America 2004;13:113-27.

2. Guzzo M, Locati LD, Prott FJ, et al. Major and minor salivary gland tumors. Crit Rev Oncol Hematol 2010;74:134-48.

3. Andry G, Hamoir M, Locati LD, et al. Management of salivary gland tumors. Expert Rev Anticancer Ther 2012;12:1161-8.

4. Orlandi E, Iacovelli NA, Bonora M, et al. Salivary Gland. Photon beam and particle radiotherapy: Present and future. Oral Oncol 2016;60:146-56.

5. Safdieh J, Givi B, Osborn V, et al. Impact of Adjuvant Radiotherapy for Malignant Salivary Gland Tumors. Otolaryngol Head Neck Surg 2017;157:988-94.

6. Kaur J, Goyal S, Muzumder S, et al. Outcome of surgery and post-operative radiotherapy for major salivary gland carcinoma: ten year experience from a single institute. Asian Pac J Cancer Prev 2014;15:8259-63.

7. Hosni A, Huang SH, Goldstein D, et al. Outcomes and prognostic factors for major salivary gland carcinoma following postoperative radiotherapy. Oral Oncol 2016;54:75-80.

8. Iqbal H, Bhatti AB, Hussain R, et al. Ten year experience with surgery and radiation in the management of malignant major salivary gland tumors. Asian Pac J Cancer Prev 2014;15:2195-9.
9. Al-Mamgani A, van Rooij P, Verduijn GM, et al. Longterm outcomes and quality of life of 186 patients with primary parotid carcinoma treated with surgery and radiotherapy at the Daniel den Hoed Cancer Center. Int J Radiat Oncol Biol Phys 2012;84:189-95.

10. Stannard C, Vernimmen F, Carrara H, et al. Malignant salivary gland tumours: can fast neutron therapy results point the way to carbon ion therapy? Radiother Oncol 2013;109:262-8.

11. Douglas JG, Koh WJ, Austin-Seymour M, et al. Treatment of salivary gland neoplasms with fast neutron radiotherapy. Arch Otolaryngol Head Neck Surg 2003;129:944-8.

12. Davis C, Sikes J, Namaranian P, et al. Neutron Beam Radiation Therapy: An Overview of Treatment and Oral Complications When Treating Salivary Gland Malignancies. J Oral Maxillofac Surg 2016;74:830-5.

13. Jensen AD, Poulakis M, Nikoghosyan AV, et al. High-LET radiotherapy for adenoid cystic carcinoma of the head and neck: 15 years' experience with raster-scanned carbon ion therapy. Radiother Oncol 2016;118:272-80.

14. Sulaiman NS, Demizu Y, Koto M, et al. Multicenter Study of Carbon-Ion Radiation Therapy for Adenoid Cystic Carcinoma of the Head and Neck: Subanalysis of the Japan Carbon-Ion Radiation Oncology Study Group (J-CROS) Study (1402 HN). Int J Radiat Oncol Biol Phys 2018;100:639-46.

15. Rackwitz T, Debus J. Clinical applications of proton and carbon ion therapy. Semin Oncol 2019;46:226-32.

16. Jiang GL. Particle therapy for cancers: a new weapon in radiation therapy. Frontiers of Medicine 2012;6:165-72.

17. Grant SR, Grosshans DR, Bilton SD, et al. Proton versus conventional radiotherapy for pediatric salivary gland tumors: Acute toxicity and dosimetric characteristics. Radiother Oncol 2015;116:309-15.

18. Swisher-McClure S, Teo BK, Kirk M, et al. Comparison of Pencil Beam Scanning Proton- and Photon-Based Techniques for Carcinoma of the Parotid. Int J Part Ther 2016;2:525-32.

19. Jensen AD. Raster-scanned carbon ion therapy for malignant salivary gland tumors: acute toxicity and initial treatment response. Radiat Oncol 2011;6:149.

20. Adeberg S, Windisch P, Ehret F, et al. Intensity Modulated Radiotherapy (IMRT) With Carbon Ion Boost in the Multimodal Treatment of Salivary Duct Carcinoma. Front Oncol 2019;9:1420.

21. Nieder C, Milas L, Ang KK. Tissue tolerance to reirradiation. Semin Radiat Oncol 2000;10:200-9.

22. Emami B, Lyman J, Brown A, et al. Tolerance of normal 
tissue to therapeutic irradiation. Int J Radiat Oncol Biol Phys 1991;21:109-22.

23. Chen AM, Yoshizaki T, Velez MA, et al. Tolerance of the Brachial Plexus to High-Dose Reirradiation. Int J Radiat Oncol Biol Phys 2017;98:83-90.

24. Mendenhall WM, Morris CG, Amdur RJ, et al. Radiotherapy alone or combined with surgery for adenoid cystic carcinoma of the head and neck. Head Neck 2004;26:154-62.

25. Spratt DE, Salgado LR, Riaz N, et al. Results of photon radiotherapy for unresectable salivary gland tumors: is neutron radiotherapy's local control superior? Radiology and Oncology 2014;48:56-61.

26. Mendenhall WM, Morris CG, Amdur RJ, et al. Radiotherapy alone or combined with surgery for salivary gland carcinoma. Cancer 2005;103:2544-50.

27. Timoshchuk MA, Dekker P, Hippe DS, et al. The efficacy of neutron radiation therapy in treating salivary gland malignancies. Oral Oncol 2019;88:51-7.

28. Lee A, Givi B, Osborn VW, et al. Patterns of care and survival of adjuvant radiation for major salivary adenoid cystic carcinoma. Laryngoscope 2017;127:2057-62 .

29. Hsieh CE, Lee LY, Chou YC, et al. Nodal failure patterns and utility of elective nodal irradiation in submandibular gland carcinoma treated with postoperative radiotherapy a multicenter experience. Radiat Oncol 2018;13:184.

30. Chung MP, Tang C, Chan C, et al. Radiotherapy for nonadenoid cystic carcinomas of major salivary glands. American Journal of Otolaryngology 2013;34:425-30.

Cite this article as: Huang Q, Hu W, Hu J, Gao J, Yang J, Qiu X, Kong L, Lu JJ. Intensity-modulated proton and carbon-ion radiation therapy in the management of major salivary gland carcinomas. Ann Transl Med 2022;10(22):1195. doi: 10.21037/ atm-20-7988
31. Hayashi K, Koto M, Demizu Y, et al. A retrospective multicenter study of carbon-ion radiotherapy for major salivary gland carcinomas: Subanalysis of J-CROS 1402 HN. Cancer Sci 2018;109:1576-82.

32. Koto M, Hasegawa A, Takagi R, et al. Definitive carbonion radiotherapy for locally advanced parotid gland carcinomas. Head Neck 2017;39:724-9.

33. Ali S, Bryant R, Palmer FL, et al. Distant Metastases in Patients with Carcinoma of the Major Salivary Glands. Ann Surg Oncol 2015;22:4014-9.

34. Mariano FV, da Silva SD, Chulan TC, et al. Clinicopathological factors are predictors of distant metastasis from major salivary gland carcinomas. International Journal of Oral and Maxillofacial Surgery 2011;40:504-9.

35. Ogata T, Teshima T, Kagawa K, et al. Particle irradiation suppresses metastatic potential of cancer cells. Cancer Res 2005;65:113-20.

36. Matsumoto Y, Furusawa Y, Uzawa A, et al. Antimetastatic Effects of Carbon-Ion Beams on Malignant Melanomas. Radiat Res 2018;190:412-23.

37. Romesser PB, Cahlon O, Scher E, et al. Proton beam radiation therapy results in significantly reduced toxicity compared with intensity-modulated radiation therapy for head and neck tumors that require ipsilateral radiation. Radiother Oncol 2016;118:286-92.

(English Language Editor: D. Fitzgerald) 